\title{
Gastrointestinal tract metastasis from tubulolobular carcinoma of the breast: a case report and review of the literature
}

This article was published in the following Dove Press journal:

OncoTargets and Therapy

18 March 2014

Number of times this article has been viewed

\section{Guixin Wang \\ Tingjiang Wang \\ Jian Jiang \\ Luyao Zhou \\ Haidong Zhao}

Department of Breast Surgery, The Second Affiliated Hospital of Dalian Medical University, Shahekou District, Dalian, Liaoning Province, People's Republic of China
Correspondence: Haidong Zhao

The Second Affiliated Hospital of Dalian Medical University, Zhongshan Road No 467, Shahekou District, Dalian, Liaoning Province, People's Republic of China Tel +86I 5940836673

Email z.hddl@hotmail.com
Abstract: Metastasis of breast cancer into the gastrointestinal tract happens rarely. The diagnosis of this kind of disease is difficult because of the nonspecific symptoms and the long interval between primary manifestations and recurrence. Awareness of this condition may lead to an accurate diagnosis and an earlier initiation of systemic treatment, thus avoiding unnecessary surgical intervention. In this paper, we report a rare case of a patient with tubulolobular carcinoma metastases to the colon, presenting with abdominal pain, discomfort, and weight loss. The patient underwent radical mastectomy and received postoperative radiotherapy and chemotherapy. Ten years later, she presented with gastrointestinal tract symptoms. Surgery combined with systemic treatment was chosen for the colon lesion. Immunohistochemical staining suggested a breast origin. The patient was still living 24 months after the diagnosis of the metastasis. This is the fourth case report in our literature review.

Keywords: breast cancer, tubulolobular carcinoma, metastasis, gastrointestinal tract

\section{Introduction}

Breast cancer $(\mathrm{BC})$ is the most common malignant tumor, accounting for significant cause of death in females. The common pathologic types, ductal and lobular carcinoma, can be distinguished by the typical cytologic and architectural criteria, but some carcinoma, such as tubulolobular carcinoma, can display a mixture of ductal and lobular carcinoma-like cells. As with other types of BC, tubulolobular carcinoma usually metastasizes to lymph nodes, bone, lung, liver, or brain, but occasionally can affect the gastrointestinal (GI) tract.

In this paper, we report a rare case of a patient with tubulolobular carcinoma of the breast with metastases to the colon, presenting with abdominal pain, discomfort, and weight loss.

\section{Case report}

A 70-year-old woman was admitted to our hospital with pain and discomfort in the right side of the abdomen, weight loss over the preceding 10 months, and tenderness of a right abdominal mass that had begun to develop 3 months before. Ten years before, she had received a left radical mastectomy as well as a dissection of axillary lymph nodes. Histopathological examination suggested lobular infiltrating carcinoma and some of the signet ring cell carcinoma (Figure 1A). Metastases were identified in five out of eight left axillary lymph nodes, but were not found in the left greater pectoral muscle. Immunohistochemical stains showed tumor cells CK7 (+), GCDFP-15 (gross cystic disease fluid Protein-15) (+), and c-erbB2 (++). The final histological stage was 



Figure I The pathological images of primary breast cancer and the colonic mass.

Notes: (A) Mastectomy specimen obtained in 200I, showing lobular infiltrating carcinoma, are similar to infiltrating ductal carcinoma (hematoxylin and eosin stain, $\times 100$ ). (B) Right-colectomy specimen obtained in 2011, showing poorly differentiated ductal adenocarcinoma. Tumor emboli can be found in some lymph vessels (hematoxylin and eosin stain, $\times 100)$.

assessed as IIIa (pT3N1M0) (NCCN clinical practice guidelines of breast cancer). After surgery, the patient received one cycle of adjuvant chemotherapy, but this was discontinued due to serious GI reaction. Then, she received treatment with capecitabine (2,000 mg orally twice daily on days 1-14 [21day cycle] from March 2001 until March 2002) and radiation therapy at the left clavicle $\left({ }^{60} \mathrm{Co} \mathrm{DT:50Gy/38f)}\right.$, the left internal mammary (12Mev DT:50Gy/32f), and the chest wall (6Mev DT:50Gy/32f). After radiation therapy, she received tamoxifen but this was discontinued due to severe endometrial thickening after 3 months. Before chemotherapy, further immunohistochemical stains performed by the oncology department showed E-cadherin (+), 34ßE12 (-), ER (+), PR (+), CK19 $(+)$, CK20 (+), suggesting the presence of ductal infiltrating carcinoma and not lobular infiltrating carcinoma.

Ten years later, she complained of pain and a hard mass with tenderness at the right side of her abdomen. A color Doppler ultrasound showed a $5.0 \times 4.9 \mathrm{~cm}$, isoechoic, and irregularly shaped mass near the second hepatic portal in right lobe of the liver and a $7.5 \times 7.0 \times 5.5 \mathrm{~cm}$, low-echoic, and irregularly shaped mass at the right side of the abdomen. Abdominal magnetic resonance imaging (MRI) demonstrated space-occupying lesions on the anterior section of the luminal side of the mid-ascending colon and the right lobe of the liver. The enhanced computed tomography (CT) scan, after admission, also showed a $5.0 \times 4.9$ $\mathrm{cm}$, low-density, oval, and sub-lobe opacity mass, which could be ring-enhanced in the right lobe of the liver, and a $7.6 \times 5.9 \mathrm{~cm}$ soft tissue mass with density in the luminal wall of the ascending colon (Figure 2). Cancer antigen (CA)-125 levels were significantly high, at $365.5 \mathrm{U} / \mathrm{mL}$, while CEA, CA15-3, and CA19-9 levels were normal. Because of the clinical symptoms, a right colectomy was performed after admission. In the operation, a $10-15 \mathrm{~cm}$ mass at the hepatic flexure of the ascending colon that had projected into the lumen instead of breaking through the serosa (or serous membrane), was found. Abnormal mesenteric lymph node was not found. The surgeon suspected there was a primary colon cancer. Histopathological examination showed poorly differentiated adenocarcinoma that had infiltrated into all the layers of colonic wall from the serosa, and cancer emboli were found in colonic lymph vessels (Figure 1B). Immunohistochemical stains showed tumor cells $\mathrm{CK} 7(+)$, E-cadherin (+), 34ßE12 (-), ER (+), GCDFP-15 (+), CK19(+), CK20 (+), and liver cell (-) (Figure 3). These results confirmed that the mass was metastatic ductal infiltrating carcinoma. The patient underwent transcatheter arterial embolization. She rejected intravenous administration (navelbine and cisplatin [NP] regimen) but received treatment with capecitabine $(2,000 \mathrm{mg}$ orally twice daily on days 1-14 [21-day cycle]) from December 2011 until February 2013. The symptoms were significantly relieved, and the patient was still living 24 months after diagnosis of the metastasis and at the time this paper was written.

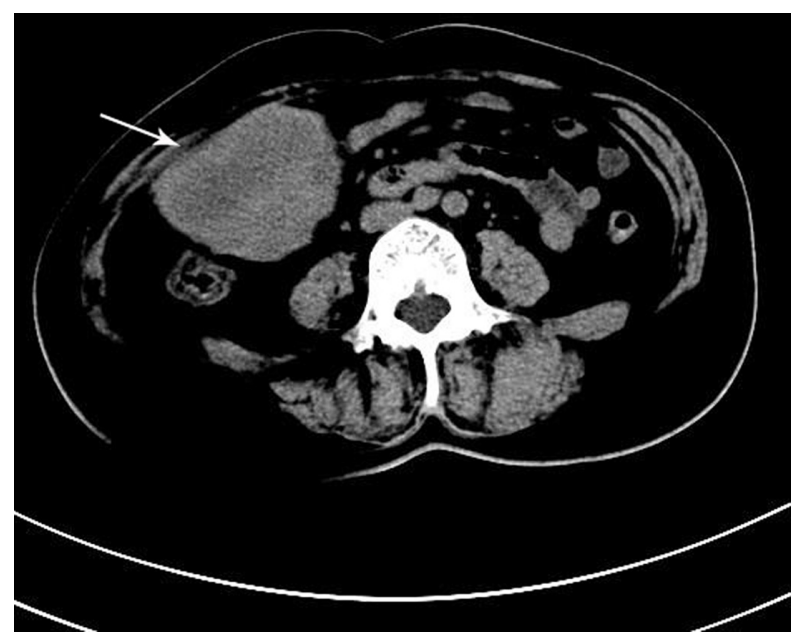

Figure 2 Abdominal computed tomography scan demonstrates an obvious thickened ascending colon and a soft-tissue mass (arrow) measuring $7.6 \times 5.9 \mathrm{~cm}$ with a 3I Hounsfield unit $(\mathrm{HU})$ computed tomography value. 


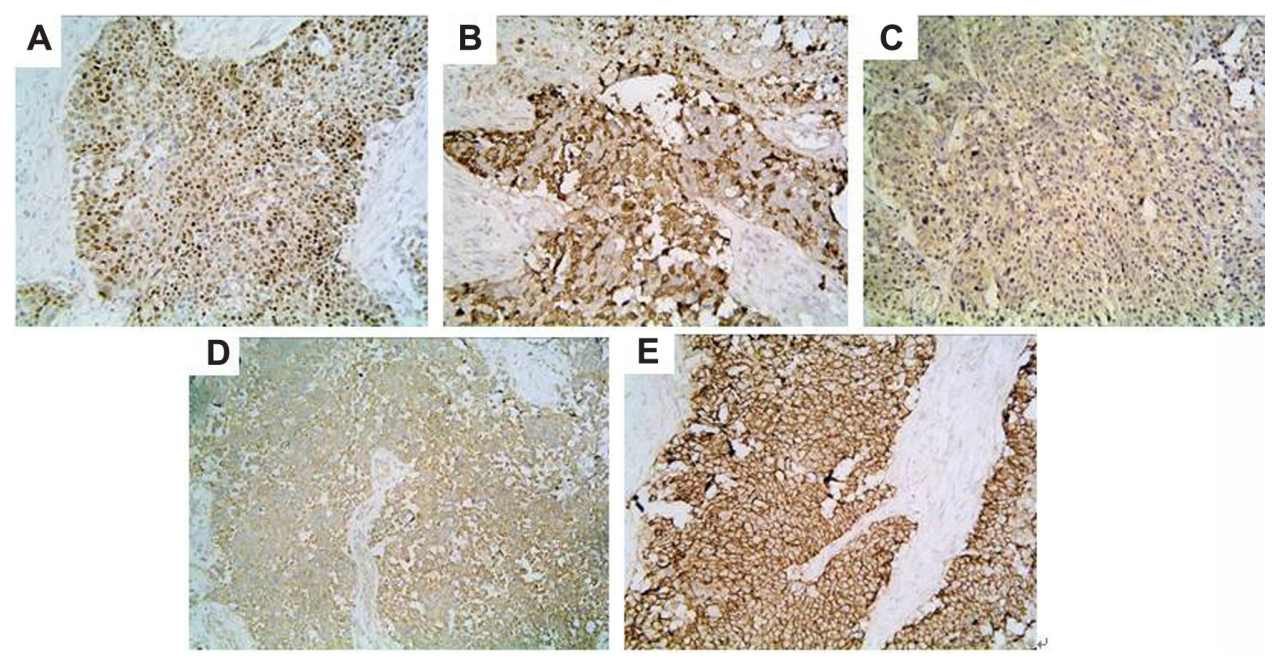

Figure 3 Immunohistochemistry scan of right-colectomy specimen.

Notes: (A) ER (+), ×200; (B) CK7 (+), ×200; (C) CK20 (+), ×200; (D) GCDFP-I5 (+), ×200; (E) E-cadherin (+), ×200.

Abbreviations: ER, estrogen receptor; CK7, cytokeratin 7; CK20, cytokeratin 20; GCDFP-I5, gross cystic disease fluid Protein-I5.

\section{Discussion}

$\mathrm{BC}$ is the most common malignant tumor and accounts for a large number of cancer-related deaths ${ }^{1}$ in women. In the last few decades, several advances in the treatment of $\mathrm{BC}$ have significantly improved the survival rate and decreased mortality ${ }^{2}$ however, the prognosis for the systemic recurrence of adenocarcinomas remains difficult to assess, as they can occur in any organ, mainly the lymph nodes, lungs, pleura, bones, and liver. ${ }^{3} \mathrm{GI}$ metastases from $\mathrm{BC}$ are rare. It has been reported that occurrence of GI metastases are less than $1 \%$ in all subsequent metastatic $\mathrm{BC},{ }^{4,9-11}$ and that colorectal metastases are even rarer, with only a few cases having been reported. ${ }^{5}$

$\mathrm{As} \mathrm{BC}$ is a heterogeneous group of tumors with various genetic and biological features, some studies have also suggested an association between the metastasized organs and the pathological types of BC. Compared to lobular infiltrating carcinoma, infiltrating ductal carcinoma seems to spread less frequently to the GI tract. In a study of 2,605 patients, the metastatic rate of infiltrating ductal carcinoma to the GI tract was considerably lower than that of lobular infiltrating carcinoma $(0.2 \%$ versus $4.5 \% ; P<0.05) .{ }^{4}$ The mechanism behind this correlation requires further studies.

The literature reporting cases of colorectal metastasis are summarized in Table 1. In these cases, the most common clinical symptom was alteration in bowel habit. There are also some reports of cases presenting other symptoms of bowel stenosis, such as abdominal pain, and systemic symptoms, such as weight loss or anemia. Therefore, a differential diagnosis between a metastatic $\mathrm{BC}$ and a primary GI cancer is difficult to make because the symptoms of metastatic $\mathrm{BC}$ in the GI tract are similar to those of primary colorectal tumors, Crohn's disease, and several other benign tumor processes. Moreover, a long, asymptomatic interval between first diagnosis and metastasis can also confuse the diagnosis. From our review of the literature, this interval could often be more than 4 years, and even as long as 28 years. ${ }^{6}$ In such studies, diagnosis of metastasis depends on screening during the follow-up period. Recently, a large systemic review recommended regular history including, physical examination, and mammography for BC follow-up, although the use of blood tests, chemistry panels, bone scans, chest radiographs, ultrasounds, CT, MRI, positron emission tomography-CT, and tumor markers were not recommended in an otherwise asymptomatic patient with no specific findings on clinical examination. ${ }^{7}$ Endoscopy with biopsy may mislead diagnosis if the specimens are taken superficially, while metastases often appear in the muscularis propria layer without endothelial changes. ${ }^{4}$ Although CT and MRI have a high spatial resolution and can give excellent anatomical images, it is very hard for them to differentiate primary tumor from metastasis, necroses, or scars. Moreover, plasma CA-125 levels were obviously elevated in our case, while CEA, CA15-3, and CA19-9 levels were normal. While an elevated level of CA-125 may alert clinicians to the possibility of metastatic $\mathrm{BC}$, it is not a highly specific tumor maker that allows for distinction between $\mathrm{BC}$ metastases, colorectal metastases, and other tumor metastases.

In our case, the patient did not undergo a standard follow-up evaluation until symptoms occurred, which was when the metastatic tumor had already reached a remarkable size. In addition, it has been reported that second primary cancers are more common than colorectal metastases in patients with a history of $\mathrm{BC} .^{8}$ Therefore, we decided to 


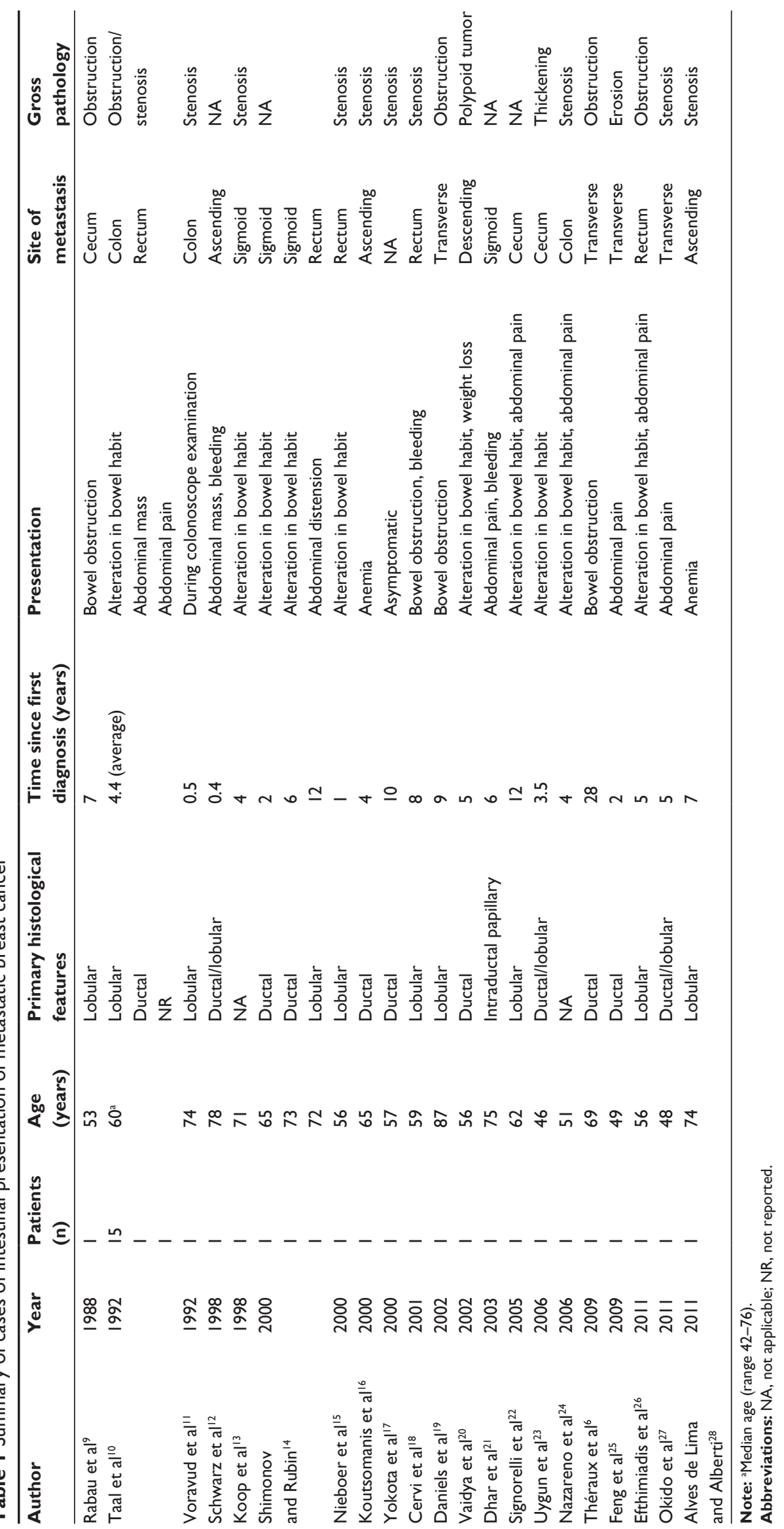


do an open laparotomy to relieve the clinical symptoms first, and then make a definitive diagnosis by pathology. Although this patient survived and has enjoyed a high quality of life after operation, utilization of some invasive approaches, such as endoscopy or aparoscopy. Laparoscopy, may be more helpful for diagnosis. The final diagnosis still should depend on histopathology. Although histopathological observation is often used to distinguish ductal from lobular carcinomas, the first and second results were contradictory. The patient may encounter tubulolobular carcinoma, a rare variant carcinoma that displays histologic features of both ductal and lobular carcinomas, first reported by Fisher et $\mathrm{al}^{29}$ and about which only three case reports were published before the present case. ${ }^{11,22,26}$ A recent study also demonstrated that metastases of tubulolobular carcinoma could predominantly show tubules. ${ }^{30}$ Therefore, immunohistochemical staining has proved very helpful to the diagnosis. As BC is often derived from epithelium, CK7, CK19, and CK20, which are the biomarkers of epithelial tumors, can usually be positive both in primary $\mathrm{BC}$ and metastases, but they cannot be used in differentiating $\mathrm{BC}$ from other epithelial tumors. ${ }^{31-33}$ E-cadherin protein, which is often negative in lobular carcinomas and positive in ductal carcinomas, is also a useful diagnostic tool for differentiating ductal from lobular carcinomas. ${ }^{34}$ E-cadherin can also display membranous staining in tubulolobular carcinomas. ${ }^{30}$ In addition, ER, PR, and GCDFP-15 are all highly specific for metastatic breast carcinomas; they may be positive in both primary $\mathrm{BC}$ and metastatic lesions, ${ }^{35}$ while $34 \beta \mathrm{E} 12$ is often negative. ${ }^{36}$

In the present case, the results of immunohistochemical staining were strong evidence for the diagnosis of metastatic $\mathrm{BC}$, even though ER, PR, and c-erbB2 were all positive. Treatment protocols for such cases remain controversial. Surgical excision of metastases is usually necessary for an exact diagnosis or relieving serious clinical manifestations such as perforation, hemorrhage, or bowel obstruction. Some studies have reported that patients could achieve long-term survival after radical surgery to remove a solitary metastasis. ${ }^{16,37}$ Although extensive metastasis cannot be detected in preoperative investigations, systemic treatment (chemotherapy, endocrine treatment, or both) is usually utilized in patients with GI metastases, since patients usually present with involvement of multiple organs. Treatment combining surgery and systemic treatment is often effective, ${ }^{18}$ particularly in those patients that present no other extensive metastases. ${ }^{16,20}$

Despite advances in treatment, prognosis of GI metastases is still poor and few patients survive beyond 2 years. It was reported that the mean survival of patients diagnosed with
GI tract metastasis was 12 to 16 months; ${ }^{1,12}$ however, there is still a lack of large clinical trials regarding the surgical or systemic treatment of BC metastasis to the colon. In our case, surgery combined with systemic treatment to treat the colonic metastasis gave a satisfactory result.

\section{Conclusion}

GI tract metastasis from BC is very rare, and accurate and definite diagnosis is difficult due to nonspecific clinical presentations and long intervals between manifestations. Regular follow-up, physical examination, and mammography are recommended to make an early diagnosis and to inform the choice of the correct therapeutic approach. The history of $\mathrm{BC}$ and immunohistochemical staining of biopsies, compared with original $\mathrm{BC}$, can help with correct diagnosis. As most patients with colonic metastases may have widespread metastases, systemic treatment is recommended as the firstline treatment. Surgery combined with systemic treatment is also effective, especially for patients with significant clinical manifestations.

\section{Disclosure}

The authors report no conflicts of interest in this work.

\section{References}

1. Taal BG, Peterse H, Boot H. Clinical presentation, endoscopic features, and treatment of gastric metastases from breast carcinoma. Cancer. 2000;89(11):2214-2221.

2. Chu KC, Tarone RE, Kessler LG, et al. Recent trends in US breast cancer incidence, survival, and mortality rates. J Natl Cancer Inst 1996;88(21):1571-1579.

3. Lee YT. Breast carcinoma: pattern of metastasis at autopsy. J Surg Oncol. 1983;23(3):175-180.

4. Borst MJ, Ingold JA. Metastatic patterns of invasive lobular versus invasive ductal carcinoma of the breast. Surgery. 1993;114(4):637-641; discussion 641-632.

5. Ellis IO, Galea M, Broughton N, Locker A, Blamey RW, Elston CW. Pathological prognostic factors in breast cancer. II. Histological type. Relationship with survival in a large study with long-term follow-up. Histopathology. 1992;20(6):479-489.

6. Théraux J Theraux J, Bretagnol F, Guedj N, Cazals-Hatem D, Panis Y. Colorectal breast carcinoma metastasis diagnosed as an obstructive colonic primary tumor. A case report and review of the literature. Gastroenterol Clin Biol. 2009;33(12):1114-1117.

7. Khatcheressian JL, Hurley P, Bantug E, et al; American Society of Clinical Oncology. Breast cancer follow-up and management after primary treatment: American Society of Clinical Oncology clinical practice guideline update. J Clin Oncol. 2013;31(7):961-965.

8. Agarwal N, Ulahannan MJ, Mandile MA, Cayten CG, Pitchumoni CS Increased risk of colorectal cancer following breast cancer. Ann Surg 1986;203(3):307-310.

9. Rabau MY, Alon RJ, Werbin N, Yossipov Y. Colonic metastases from lobular carcinoma of the breast. Report of a case. Dis Colon Rectum. 1988;31(5):401-402.

10. Taal BG, den Hartog Jager FC, Steinmetz R, Peterse H. The spectrum of gastrointestinal metastases of breast carcinoma: II. The colon and rectum. Gastrointest Endosc. 1992;38(2):136-141. 
11. Voravud N, el-Naggar AK, Balch CM, Theriault RL. Metastatic lobular breast carcinoma simulating primary colon cancer. Am J Clin Oncol. 1992;15(4):365-369.

12. Schwarz RE, Klimstra DS, Turnbull AD. Metastatic breast cancer masquerading as gastrointestinal primary. Am J Gastroenterol. 1998;93(1):111-114.

13. Koop H, Dombrowski H, Maroske D, Schwerk WB, Schmitz-Moormann P, Arnold R. [Segmental colonic stenosis in intestinal metastasis of breast carcinoma. A contribution to the differential diagnosis of colitis]. Dtsch Med Wochenschr. 1988;113(27):1101-1104. German.

14. Shimonov M, Rubin M. Metastatic breast tumors imitating primary colonic malignancies. Isr Med Assoc J. 2000;2(11):863-864.

15. Nieboer P, van der Graaf WT, de Knegt RJ, van Dullemen HM. Rectal syndrome as first presentation of metastatic breast cancer. Am J Gastroenterol. 2000;95(8):2138-2139.

16. Koutsomanis D, Renier JF, Ollivier R, Moran A, el-Haite AA. Colonic metastasis of breast carcinoma. Hepatogastroenterology. 2000;47(33): 681-682.

17. Yokota T, Kunii Y, Kagami M, et al. Metastatic breast carcinoma masquerading as primary colon cancer. Am J Gastroenterol. 2000;95(10): 3014-3016.

18. Cervi G, Vettoretto N, Vinco A, et al. Rectal localization of metastatic lobular breast cancer: report of a case. Dis Colon Rectum. 2001;44(3): 453-455.

19. Daniels IR, Layer GT, Chisholm EM. Bowel obstruction due to extrinsic compression by metastatic lobular carcinoma of the breast. J R Soc Promot Health. 2002;122(1):61-62.

20. Vaidya JS, Mukhtar H, Bryan R. Colonic metastasis from a breast cancera case report and a few questions. Eur J Surg Oncol. 2002;28(4): 463-464.

21. Dhar S, Kulaylat MN, Gordon K, Lall P, Doerr RJ. Solitary papillary breast carcinoma metastasis to the large bowel presenting as primary colon carcinoma: case report and review of the literature. Am Surg. 2003;69(9):799-803.

22. Signorelli C, Pomponi-Formiconi D, Nelli F, Pollera CF. Single colon metastasis from breast cancer: a clinical case report. Tumori. 2005;91(5):424-427.

23. Uygun K, Kocak Z, Altaner S, Cicin I, Tokatli F, Uzal C. Colonic metastasis from carcinoma of the breast that mimics a primary intestinal cancer. Yonsei Med J. 2006;47(4):578-582.

24. Nazareno J, Taves D, Preiksaitis HG. Metastatic breast cancer to the gastrointestinal tract: a case series and review of the literature. World J Gastroenterol. 2006;12(38):6219-6224.
25. Feng CL, Chou JW, Huang SF. Colonic metastasis from carcinoma of the breast presenting with colonic erosion. Endoscopy. 2009;41 Suppl 2: E276-E277.

26. Efthimiadis C, Kosmidis C, Fotiadis P, et al. Breast cancer metastatic to the rectum: a case report. Tech Coloproctol. 2011;15 Suppl 1: S91-S93.

27. Okido M, Seo M, Hamada Y, et al. Metastatic breast carcinoma simulating linitis plastica of the colon: report of a case. Surg Today. 2011;41(4): 542-545.

28. Alves de Lima DC, Alberti LR. Breast cancer metastasis to the colon. Endoscopy. 2011;43 Suppl 2 UCTN:E143-E144.

29. Fisher ER, Gregorio RM, Redmond C, Fisher B. Tubulolobular invasive breast cancer: a variant of lobular invasive cancer. Hum Pathol. 1977;8(6):679-683.

30. Esposito NN, Chivukula M, Dabbs DJ. The ductal phenotypic expression of the E-cadherin/catenin complex in tubulolobular carcinoma of the breast: an immunohistochemical and clinicopathologic study. Mod Pathol. 2007;20(1):130-138.

31. Burchill SA, Bradbury MF, Pittman K, Southgate J, Smith B, Selby P. Detection of epithelial cancer cells in peripheral blood by reverse transcriptase-polymerase chain reaction. Br J Cancer. 1995;71(2): 278-281.

32. Huang P, Wang J, Guo Y, Xie W. Molecular detection of disseminated tumor cells in the peripheral blood in patients with gastrointestinal cancer. J Cancer Res Clin Oncol. 2003;129(3):192-198.

33. Felton T, Harris GC, Pinder SE, et al. Identification of carcinoma cells in peripheral blood samples of patients with advanced breast carcinoma using RT-PCR amplification of CK7 and MUC1. Breast. 2004;13(1): $35-41$.

34. Gamallo C, Palacios J, Suarez A, et al. Correlation of E-cadherin expression with differentiation grade and histological type in breast carcinoma. Am J Pathol. 1993;142(4):987-993.

35. O'Connell FP, Wang HH, Odze RD. Utility of immunohistochemistry in distinguishing primary adenocarcinomas from metastatic breast carcinomas in the gastrointestinal tract. Arch Pathol Lab Med. 2005;129(3):338-347.

36. Jarasch ED, Nagle RB, Kaufmann M, Maurer C, Bocker WJ. Differential diagnosis of benign epithelial proliferations and carcinomas of the breast using antibodies to cytokeratins. Hum Pathol. 1988; 19(3):276-289.

37. Clavien PA, Laffer U, Torhost J, Harder F. Gastro-intestinal metastases as first clinical manifestation of the dissemination of a breast cancer. Eur J Surg Oncol. 1990;16(2):121-126.
OncoTargets and Therapy

\section{Publish your work in this journal}

OncoTargets and Therapy is an international, peer-reviewed, open access journal focusing on the pathological basis of all cancers, potential targets for therapy and treatment protocols employed to improve the management of cancer patients. The journal also focuses on the impact of management programs and new therapeutic agents and protocols on
Dovepress

patient perspectives such as quality of life, adherence and satisfaction The manuscript management system is completely online and includes a very quick and fair peer-review system, which is all easy to use. Visit http://www.dovepress.com/testimonials.php to read real quotes from published authors. 